Sudhir Bhandari ${ }^{1}$, Ajit Singh Shaktawat ${ }^{1}$, Amit Tak ${ }^{2}$, J Jyotsna Shukla ${ }^{3}$, Jitentdra Gupta ${ }^{3}$, Bhoopendra Patel ${ }^{4}$, Shivankan Kakkar ${ }^{5}$, Amitabh Dube ${ }^{3}$, Sunita Dia ${ }^{6}$, Mahendra Dia7, Todd C Wehner ${ }^{7}$

${ }^{1}$ Department of Medicine, SMS Medical College and Hospitals, Jaipur, Rajasthan, India

${ }^{2}$ ICMR - National Centre for Disease Informatics and Research, Bengaluru, Karnataka, India

${ }^{3}$ Department of Physiology, SMS Medical College and Hospitals, Jaipur, Rajasthan, India

${ }^{4}$ Department of Physiology, Government Medical College, Barmer, Rajasthan, India

${ }^{5}$ Department of Pharmacology, SMS Medical College and Hospitals, Jaipur, Rajasthan, India

${ }^{6}$ Department of Rheumatology, Medstar Washington Hospital Center, Washington DC, United States

${ }^{7}$ Department of Horticultural Science, North Carolina State University, Raleigh, United States

\title{
Evaluating interactions between hyperglycemia and clotting factors in patients suffering with SARS-CoV-2 infection
}

\section{ABSTRACT}

Background. With coronavirus disease-19 (COVID-19), patients with diabetes mellitus are dealing with two pandemics and are at a higher risk of mortality. The present study was undertaken to evaluate interactions between hyperglycemia and clotting factors in COVID-19 patients.

Methods. In this retrospective observational study, 53 real-time RT-PCR SARS-CoV-2 positive cases in $\mathbf{4 0}$ to 70 years of age, representing both sexes, were enrolled in the study from SMS Medical College, Jaipur (Rajasthan, India). Based on their history of diabetes mellitus and exclusion criterion, patients were divided into diabetics $(N=11)$ and non-diabetics $(N=17)$ groups. The data on clinical profile and coagulation profile was recorded along with the markers of inflammation and infection. The two groups were compared using the Mann-Whitney test and the Fisher's exact test. Correlation coefficients between clotting factors were compared between two groups using Student $t$ test. Results. There was no significant difference in age $(p=0.25)$ or gender $(p=0.12)$ between the two groups.

Address for correspondence:

Amit Tak

ICMR - National Centre for Disease Informatics

Bengaluru, Karnataka, India

e-mail: dramitttak@gmail.com

Clinical Diabetology 2021, 10, 1: 114-122

DOI: $10.5603 /$ DK.a2021.0022

Received: 22.11.2020

Accepted: 28.12.2020
The coagulation indicators FDP $(p=0.79)$, D-dimer $(p=0.30)$, APPT $(p=0.96)$, PT $(p=0.79)$, INR $(p=1.00)$ PLT $(p=0.17)$ and the markers of inflammation and infection did not differ significantly between the two groups. There was no significant difference in correlation coefficients among coagulation indicators between the two groups ( $p>0.05$ ).

Conclusion. The study concludes that pathogenesis in clotting system is not significantly different in studied groups. Further research is needed to explain the higher mortality in diabetic patients suffering from COVID-19. (Clin Diabetol 2021; 10, 1: 114-122)

Key words: clotting factors, COVID-19, diabetes mellitus, hyperglycemia, SARS-CoV-2

\section{Introduction}

As of 21 November 2020, World Health Organization reported $57,274,018$ cases and $1,368,000$ deaths due to severe acute respiratory syndrome coronavirus 2 (SARS-CoV-2) infection for all countries of the world [1]. In 2019, International Diabetes Federation, reported that 463 million adults suffer from diabetes mellitus [2]. Diabetes mellitus is an important risk factor that causes adverse outcome in COVID-19, similar to influenza related mortality. Disease specific mortality rates due to diabetes outnumber mortality rate due to other cardiovascular diseases and cancer [3].

The molecular mechanisms that lead to diabetic complications have been studied extensively and in- 


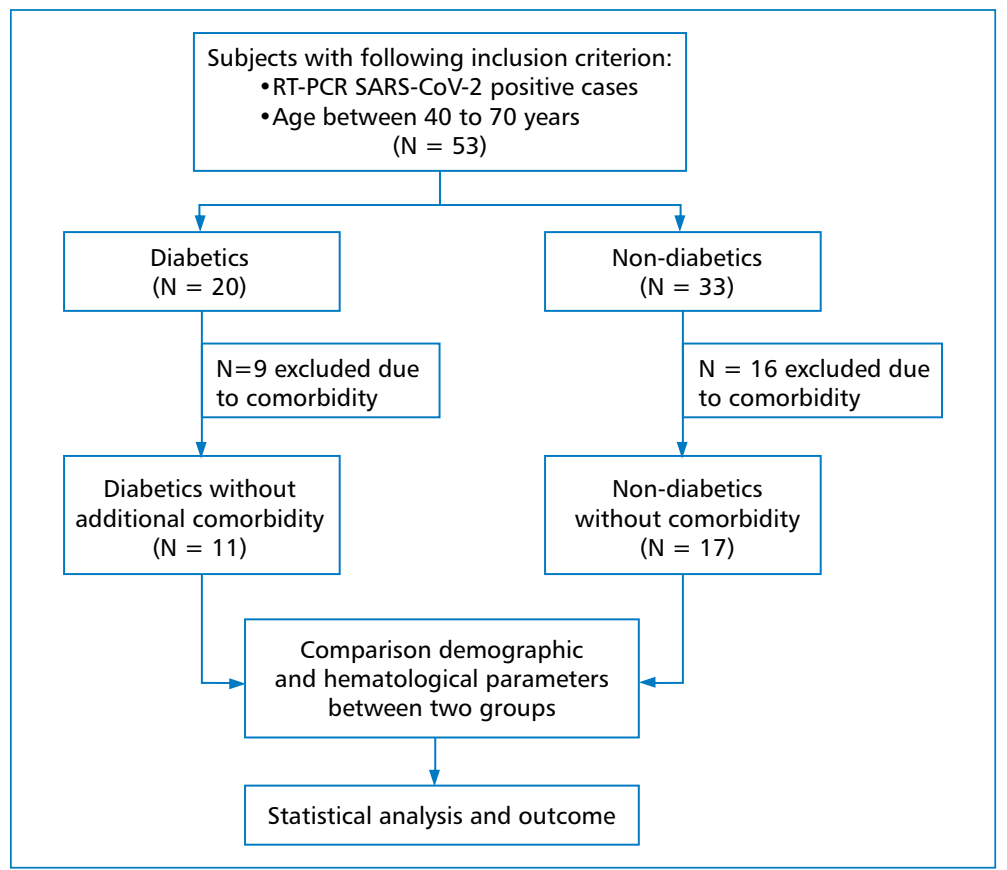

Figure 1. Flowchart of the study

volve reactive oxygen species (ROS) such as hydrogen peroxide and superoxide anions [4, 5]. These reactive species could deteriorate the cellular functions of multiple systems of the body. The pathophysiological mechanisms of COVID-19 has been explained. The angiotensin converting enzyme 2 (ACE-2) receptors are present in multiple organ systems of the body. The entry of SARS-CoV-2 via ACE-2 receptors in respiratory tract cells and pneumocytes has been mentioned. Further pathogenesis involve inflammatory response including recruitment of cells and a cytokine storm. In severe conditions, there is an activation of the coagulation cascade that can lead to thrombogenesis and disseminate intravascular coagulation resulting in death of the patient [6].

An area of prime importance is the common pathway where pathogenesis of COVID-19 and diabetes mellitus interact. Furthermore, that common pathway might be an area we can intervene therapeutically in patients with diabetes mellitus who are suffering from COVID-19. The present study is undertaken to explore the effects on coagulation and the fibrinolytic system in diabetics and non-diabetics suffering from COVID-19.

\section{Material and methods}

A hospital based retrospective observational study was planned to compare indicators of coagulation, and markers of inflammation and infection between diabetics and non-diabetics in patients suffering from
COVID-19. The study was approved by the institutional ethics committee.

\section{Participants}

We enrolled 53 patients. They all had SARS-CoV-2 (identified with real-time RT-PCR) and were 40 to 70 years of age, of either sex, obtained from SMS Medical College, Jaipur, Rajasthan, India. On the basis of history of diabetes mellitus, patients were divided into two groups: diabetics with COVID-19 ( $N=20)$ and non-diabetics with COVID-19 $(\mathrm{N}=33)$. As per our criteria, patients with co-morbidities other than diabetes mellitus were excluded. After exclusion, the diabetic group had 11 patients and the non-diabetic group has 17 patients (Figure 1).

\section{Data collection}

The age, gender and COVID-19 symptoms were recorded from case sheets. The coagulation indictors included fibrin degradation products (FDP) in mg per liter, D-dimer in mg per liter FEU, activated partial thromboplastin time (APTT) in seconds, prothrombin time (PT)in seconds, and the international normalized ratio (INR). The inflammatory mediators included erythrocyte sedimentation rate (ESR) in $\mathrm{mm}$ for the $1^{\text {st }}$ hour), ferritin (mg per liter) and procalcitonin (PCT) in mg per liter. Other hematologic indictors were recorded, including hemoglobin $(\mathrm{Hb})$ in $\mathrm{gm} \%$, total leukocyte count (TLC) in thousand cells per $\mathrm{mm}^{3}$, differential neutrophil 


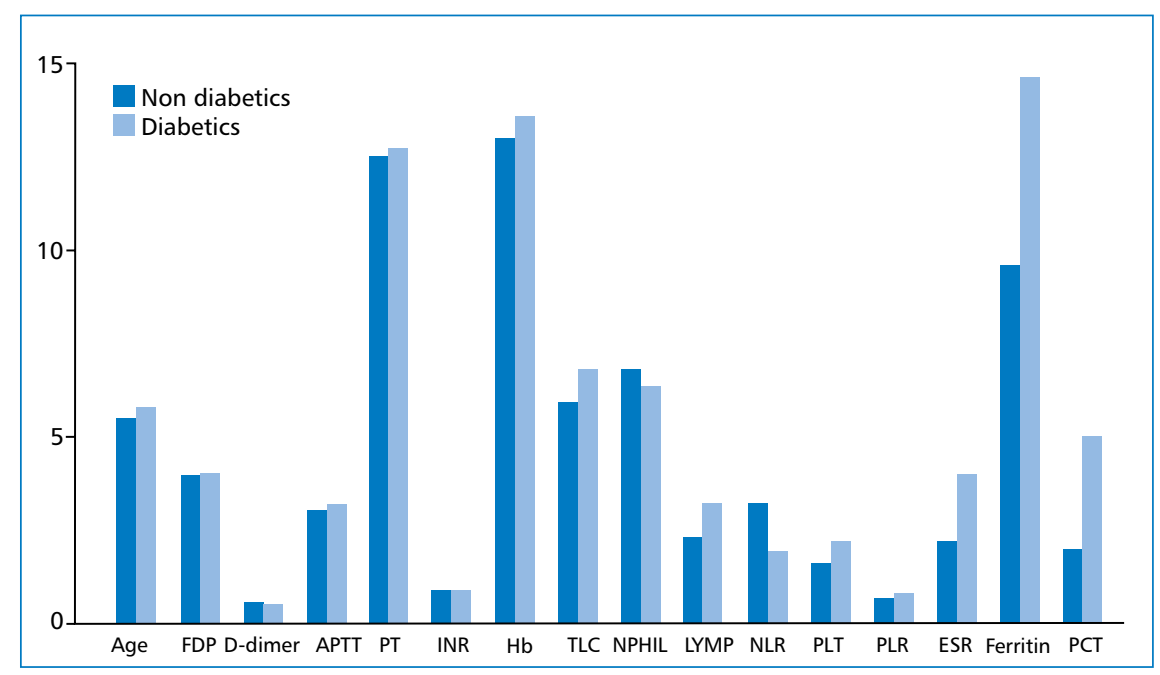

Figure 2. Comparison of medians of clinical and hematological parameters in diabetic and non-diabetic patients. Age (in $\times 10^{1}$ years); FDP - fibrin degradation products ( $\mathrm{mg}$ per litre); D-dimer ( $\mathrm{mg}$ per litre FEU; APTT — activated partial thromboplastin time ( $\times 10$ s); PT - prothrombin time (s); INR — international normalized ratio; $\mathrm{Hb}$ - hemoglobin (gm\%); TLC - total leukocyte count $\left(\times 10^{3}\right.$ cells per cubic millimeter); neutrophil - differential neutrophil count $(\times 10 \%)$; lympocyte - differential lymphocyte count ( $\times 10 \%)$; NLR - neutrophil lymphocyte ratio; PLT — platelet count ( $\times 10^{5}$ per cubic millimeter); PLR - platelet lymphocyte ratio $\left(\times 10^{-1}\right)$; ESR - erythrocyte sedimentation rate ( $\times 10$ in $1^{\text {st }}$ hour); ferritin ( $\times 10$ micrograms per liter $)$; PCT - procalcitonin $\left(\times 10^{-2}\right.$ microgram per liter)

count (\%), differential lymphocyte count (\%), platelet count (PLT) in $10^{5}$ per $\mathrm{mm}^{3}$ and C-reactive protein (CRP). Furthermore, differential neutrophil count to differential lymphocyte count ratio (NLR) and platelet count to differential lymphocyte count ratio (PLR) were calculated, and added to database.

\section{Statistical analysis}

Due to the small sample size, non-parametric analysis was performed. The quantitative variables were expressed as median ( $1^{\text {st }}$ Quartile, $3^{\text {rd }}$ Quartile) and compared with the non-parametric Mann-Whitney's test. Similarly, the qualitative attributes were expressed as proportions and compared with the two-tailed Fisher's exact test. Pearson correlations among trait pairs were calculated. Pearson correlations were contrasted between the two groups using Student t test [7] The statistical significance level was considered at 5\%. MS Excel 2010 was used to build the database and JASP (version 0.12.2) [8] and MATLAB (2016a) [9] was used for statistical analysis.

\section{Results}

The median age of diabetics (median: 58, IQR: 10.5) and non-diabetics (median: 55, IQR: 17) did not found to differ significantly $(p=0.25)$. The gender between two groups did not differ significantly $(p=0.12$ ) (Table 1 , Figure 2). The diabetic group have one patient with type $1 \mathrm{DM}(9 \%)$ and remaining patients were with type $2 \mathrm{DM}$ (91\%). The median length of hospital stay was longer in diabetic group (14.5 days) then non-diabetic group
(10 days). In diabetic group, 10 among 11 patients have been recovered (91\%) while 1 was admitted (9\%). In case of non-diabetic group, 14 cases has been recovered (82.4\%), 1 was admitted (5.8\%) and 2 died (11.8\%).

The proportion of patients with the symptoms of fever and cough were significantly higher in the diabetic group ( $p=0.02$ ). In contrast, the proportions of other symptoms such as shortness of breath (SOB) and sore throat did differ significantly between the two groups ( $p>0.05$ ) (Table 2, Figure 3).

The coagulation profile showed no significant difference with FDP $(p=0.79)$, D-Dimer $(p=0.30)$, APTT $(p=0.96)$, PT $(p=0.79)$ or INR $(p=1.00)$ between diabetics and non-diabetics (Table 1, Figure 2).

The markers of inflammation showed no significant difference in ferritin $(p=0.22)$, ESR $(p=0.08)$ or PCT $(p=0.25$ ) between diabetics and non-diabetics (Table 1 and Figure 2$)$. The CRP ( $p=0.69)$ also did not differ significantly between the two groups (Table 2 ).

Similarly, the routine hematologic parameters showed no significant difference in $\mathrm{Hb}(\mathrm{p}=0.90)$, $\operatorname{TLC}(p=0.83)$, NPHIL $(p=0.74), \operatorname{LYMP}(p=0.78)$, PLT $(p=0.17), \operatorname{NLR}(p=0.78)$ and PLR $(p=0.48)$ between diabetics and non-diabetics (Table 1, Figure 2).

The correlation coefficients among various coagulation indicators was calculated for diabetic and non-diabetic patients suffering from coronavirus disease-19. (Figures 4-6). The differences in Pearson's correlation coefficient were not significant between the two groups (Table 3). 


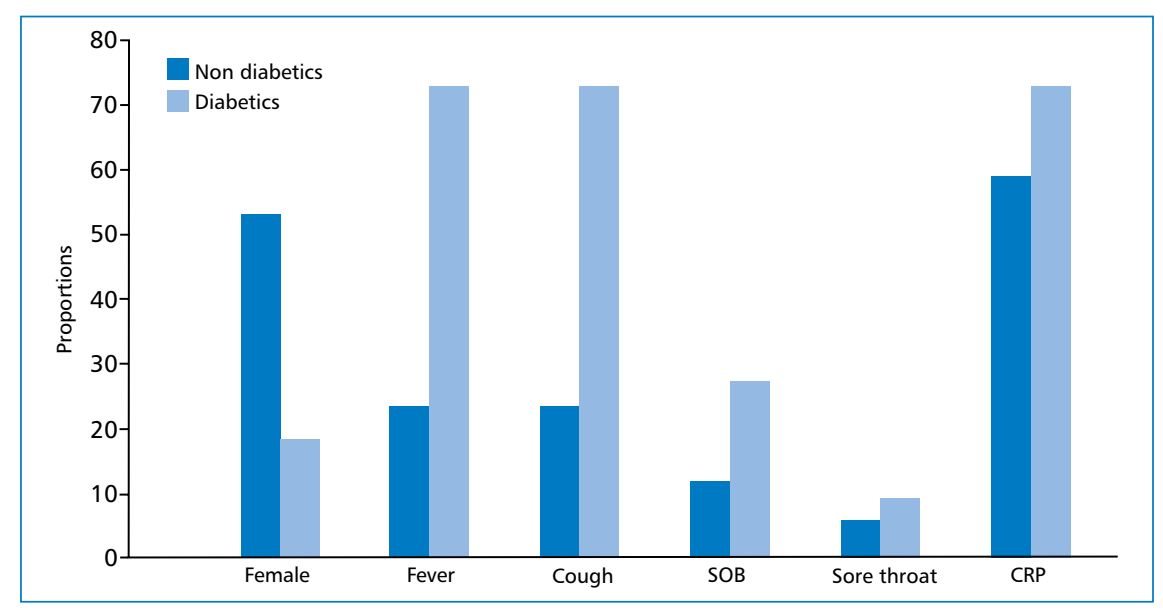

Figure 3. Comparison of proportions in clinical and hematological attributes in diabetic and non-diabetic patients. SOB - shortness of breath

Table 1. Clinical and hematological parameters in diabetic and non-diabetics patient (using non-parametric Mann Whitney test)

\begin{tabular}{|c|c|c|c|c|c|}
\hline Variables & Groups & Median & $1^{\text {st }}$ Quartile & $3^{\text {rd }}$ Quartile & $\mathbf{P}$ \\
\hline \multirow[t]{2}{*}{ Age } & Non-diabetics & 55 & 44 & 61 & 0.25 \\
\hline & Diabetics & 58 & 52 & 62.5 & \\
\hline \multirow[t]{2}{*}{ FDP } & Non-diabetics & 4 & 4 & 12.6 & 0.79 \\
\hline & Diabetics & 4 & 4 & 15.4 & \\
\hline \multirow[t]{2}{*}{ D-dimer } & Non-diabetics & 0.6 & 0.2 & 2.6 & 0.30 \\
\hline & Diabetics & 0.5 & 0.5 & 3.7 & \\
\hline \multirow[t]{2}{*}{ APTT } & Non-diabetics & 30.6 & 27.1 & 32.4 & 0.96 \\
\hline & Diabetics & 31.6 & 31.5 & 31.9 & \\
\hline \multirow[t]{2}{*}{ PT } & Non-diabetics & 12.5 & 12 & 13.5 & 0.79 \\
\hline & Diabetics & 12.7 & 11.9 & 14.55 & \\
\hline \multirow[t]{2}{*}{ INR } & Non-diabetics & 0.9 & 0.9 & 1 & 1.00 \\
\hline & Diabetics & 0.9 & 0.9 & 1.1 & \\
\hline \multirow[t]{2}{*}{$\mathrm{Hb}$} & Non-diabetics & 13 & 13 & 13.5 & 0.90 \\
\hline & Diabetics & 13.6 & 12.5 & 13.9 & \\
\hline \multirow[t]{2}{*}{ TLC } & Non-diabetics & 5.93 & 4.5 & 8.2 & 0.83 \\
\hline & Diabetics & 6.8 & 4.15 & 9.135 & \\
\hline \multirow[t]{2}{*}{ NPHIL } & Non-diabetics & 68 & 56.5 & 73.3 & 0.74 \\
\hline & Diabetics & 63.5 & 60 & 70.55 & \\
\hline \multirow[t]{2}{*}{ LYMP } & Non-diabetics & 22.9 & 20 & 35.4 & 0.78 \\
\hline & Diabetics & 32 & 21.35 & 32 & \\
\hline \multirow[t]{2}{*}{ NLR } & Non-diabetics & 3.201 & 1.54 & 3.6 & 0.78 \\
\hline & Diabetics & 1.913 & 1.875 & 3.687 & \\
\hline \multirow[t]{2}{*}{ PLT } & Non-diabetics & 1.58 & 1.4 & 2.13 & 0.17 \\
\hline & Diabetics & 2.17 & 1.605 & 2.725 & \\
\hline \multirow[t]{2}{*}{ PLR } & Non-diabetics & 0.069 & 0.031 & 0.127 & 0.48 \\
\hline & Diabetics & 0.079 & 0.05 & 0.128 & \\
\hline \multirow[t]{2}{*}{ ESR } & Non-diabetics & 22 & 15 & 44 & 0.08 \\
\hline & Diabetics & 40 & 25 & 64.5 & \\
\hline \multirow[t]{2}{*}{ Ferritin } & Non-diabetics & 96 & 68 & 280 & 0.22 \\
\hline & Diabetics & 146 & 109.45 & 535 & \\
\hline \multirow[t]{2}{*}{ PCT } & Non-diabetics & 0.02 & 0.02 & 0.04 & 0.25 \\
\hline & Diabetics & 0.05 & 0.02 & 0.072 & \\
\hline
\end{tabular}

FDP — fibrin degradation products; APTT — activated partial thromboplastin time; PT — prothrombin time; INR — international normalized ratio; Hb hemoglobin; TLC — total leukocyte count; NPHIL — neutrophil; LYMP — lymphocyte; NLR — neutrophil lymphocyte ratio; PLT — platelet lymphocyte ratio; ESR - erythrocyte sedimentation rate; PCT — procalcitonin 
Table 2. Clinical and hematological attributes in diabetic and non-diabetic patients (using two-tailed Fischer exact-test)

\begin{tabular}{lcccc}
\hline Attributes & $\begin{array}{c}\text { Non- } \\
\text {-diabetics }\end{array}$ & Diabetics & $\begin{array}{c}\text { Odds } \\
\text { ratio (OR) }\end{array}$ & P \\
\hline Female & 52.941 & 18.182 & 5.06 & 0.12 \\
Fever & 23.529 & 72.727 & 8.67 & 0.02 \\
Cough & 23.529 & 72.727 & 8.67 & 0.02 \\
SOB & 11.765 & 27.273 & 2.81 & 0.35 \\
Sore Throat & 5.882 & 9.091 & 1.60 & 1.00 \\
CRP & 58.824 & 72.727 & 1.87 & 0.69 \\
\hline
\end{tabular}

$\mathrm{SOB}$ - shortness of breath

The relationship between APTT and PT shows isolated increase of PT in both diabetics and non-diabetics (Figure 7).

\section{Discussion}

The present study is an effort to explore the effects on coagulation and the fibrinolysis cascade in diabetics and non-diabetics suffering from SARS-CoV-2 infection. As reported by Bloomgarden diabetes mellitus is an important risk factor of adverse outcome in COVID-19, as with other influenza related mortality [3]. Disease specific mortality rates due to diabetes outnumber risks due to other cardiovascular diseases and cancer.

Blood is a complex fluid and consists of plasma and formed elements - the red blood cells (RBCs), white blood cells (WBCs) and platelets. The role of coagulation and the fibrinolytic system is to prevent the blood from leaking through the blood vessels and keep the blood in liquid state respectively. The coagulation cascade involves the surface-activated intrinsic pathway, the tissue factor-activated extrinsic pathway, and the common pathway. The controlling event is the generation of thrombin, that is highly controlled dynamic process. Thrombin itself has multiple roles, both positive and negative in its production as well as removal. The metrics signifying role of various coagulation pathways has been mentioned. An isolated increase in prothrombin time (PT) indicates FVII deficiency, whereas increased APTT suggests most commonly FVIII or FXI deficiency. The prolongation of both PT and APTT suggests deficiency of FV, FX, FII, or fibrinogen abnormalities. The exposure of blood to phospholipids from damaged tissue, hemolysis, and endothelial damage lead to development of disseminated intravascular coagulation (DIC). The main mechanism is the uncontrolled production of thrombin by exposure of blood to pathologic levels of tissue factor. The most sensitive test for DIC is FDP level. The D-dimer test is more specific for detection of fibrin [10-12].

Our study showed that the coagulation and fibrinolysis system did not differ significantly in diabetic and non-diabetic patients suffering from COVID-19 (Figure 3,4 ). In addition, the markers of inflammation, including ferritin, ESR, procalcitonin and CRP did not differ significantly. The proportions of patients suffering

Table 3. Comparison of Pearson's correlation coefficients among coagulation indicators between diabetics and non-diabetics suffering from COVID-19

\begin{tabular}{|c|c|c|c|c|c|}
\hline Variable 1 & Variable 2 & $\begin{array}{l}\text { Pearson's correlation coefficient } \\
\text { (diabetics) }\end{array}$ & $\begin{array}{l}\text { Pearson's correlation coefficient } \\
\text { (non-diabetics) }\end{array}$ & Z & $\mathbf{P}$ \\
\hline FDP & D-dimer & 0.999 & 0.93 & 2.10 & $<0.05$ \\
\hline FDP & APTT & 0.207 & 0.135 & 0.07 & $>0.05$ \\
\hline FDP & PT & 0.695 & 0.008 & 0.83 & $>0.05$ \\
\hline FDP & INR & 0.761 & 0.613 & 0.28 & $>0.05$ \\
\hline FDP & PLT & -0.333 & -0.077 & -0.26 & $>0.05$ \\
\hline D-dimer & APTT & 0.21 & 0.198 & 0.01 & $>0.05$ \\
\hline D-dimer & PT & 0.694 & 0.1 & 0.74 & $>0.05$ \\
\hline D-dimer & INR & 0.757 & 0.68 & 0.16 & $>0.05$ \\
\hline D-dimer & PLT & -0.321 & -0.038 & -0.29 & $>0.05$ \\
\hline APTT & PT & -0.507 & -0.305 & -0.24 & $>0.05$ \\
\hline APTT & INR & -0.463 & 0.001 & -0.49 & $>0.05$ \\
\hline APTT & PLT & 0.05 & -0.301 & 0.35 & $>0.05$ \\
\hline PT & INR & 0.953 & 0.603 & 1.14 & $>0.05$ \\
\hline PT & PLT & -0.114 & 0.254 & -0.37 & $>0.05$ \\
\hline
\end{tabular}

FDP — fibrin degradation products; APTT — activated partial thromboplastin time; PT — prothrombin time; INR — international normalized ratio; PLT — platelet lymphocyte ratio 


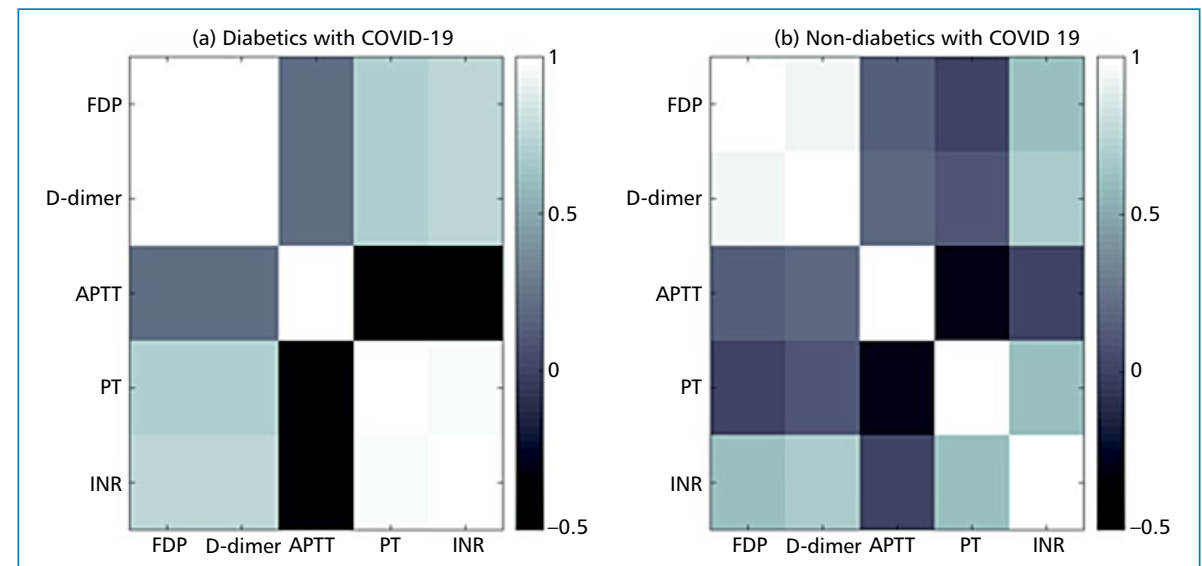

Figure 4. Correlation matrix heat maps among coagulation parameters. The color range from light to dark represents positive to negative correlation, respectively. APTT - activated partial thromboplastin time; FDP - fibrin degradation products; INR international normalized ratio; PT — prothrombin time

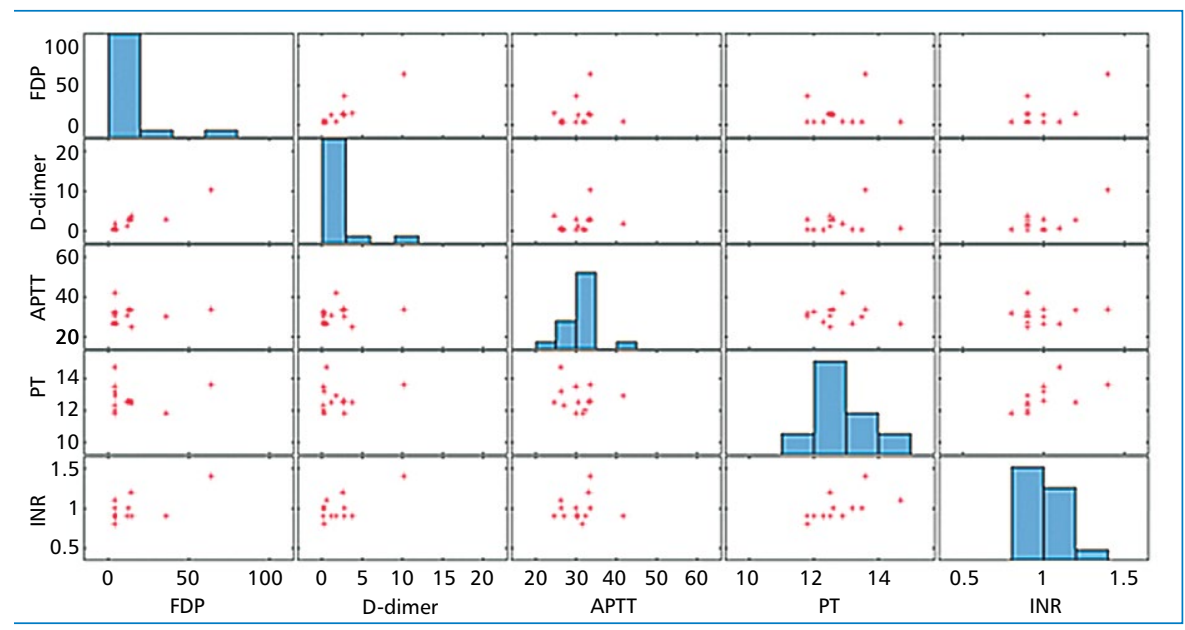

Figure 5. Correlation matrix showing correlation between coagulation indicators in non-diabetics suffering from COVID-19. APTT — activated partial thromboplastin time; FDP — fibrin degradation products; INR — international normalized ratio; PT — prothrombin time

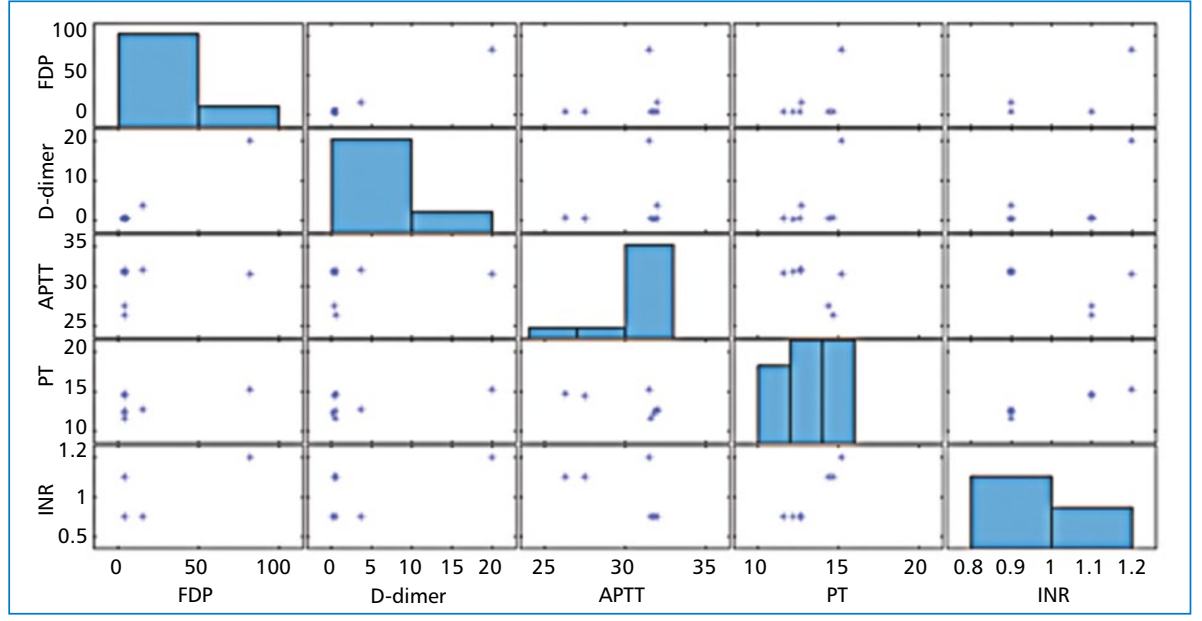

Figure 6. Correlation matrix showing correlation between coagulation indicators in diabetics suffering from COVID-19. APTT — activated partial thromboplastin time; FDP — fibrin degradation products; INR — international normalized ratio; PT — prothrombin time 


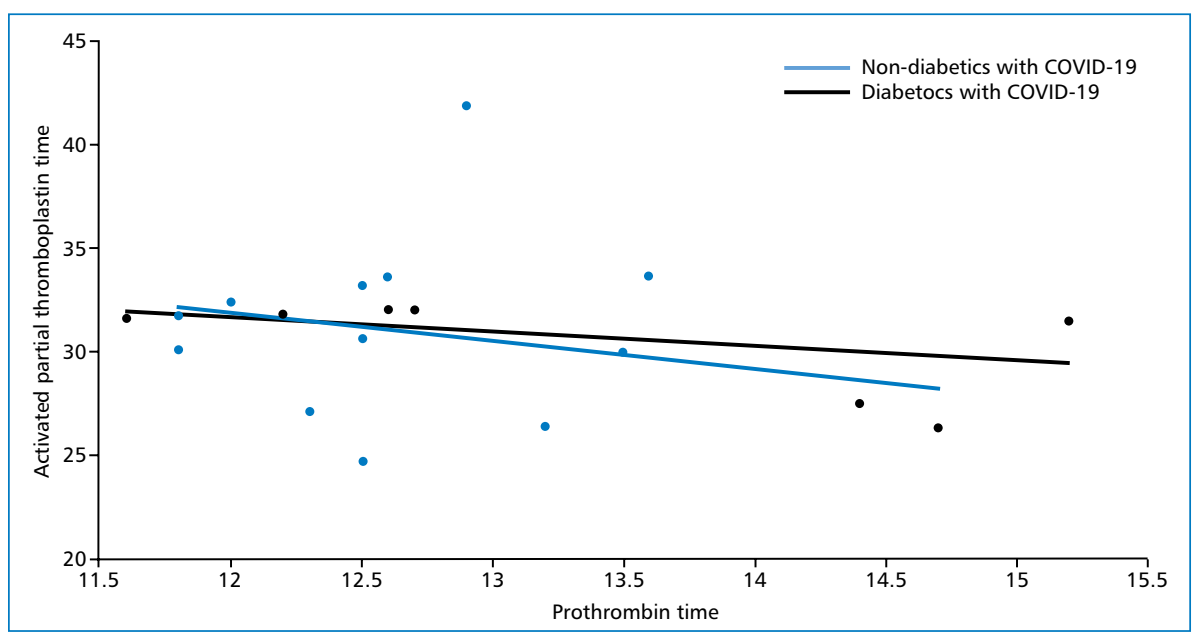

Figure 7. The correlation coefficient between prothrombin time and activated partial thromboplastin time in diabetic and nondiabetic patients

from fever and cough are higher in diabetics as compared to non-diabetics. The correlation between APPT and PT showed isolated increase in PT, suggesting role of tissue factor pathway. The difference in correlations among FDP, D-dimer, APPT, PT and INR between diabetics and non-diabetics did not differ significantly. In addition, the differential neutrophil counts and lymphocyte counts are not differ significantly in the two groups.

The pathogenesis of COVID-19 involves entry of SARS-CoV-2 into respiratory tract cells via angiotensin converting enzyme 2 receptors. Singh mentioned ACE-2 receptors expressed not only in the type I and II alveolar epithelial cells in the lungs and upper respiratory tract, but in heart, endothelium, renal tubular epithelium, intestinal epithelium, and pancreas. The binding of S-glycoprotein on the surface of SARS-CoV-2 with ACE-2 causes a conformational change in the S-glycoprotein. That lead to proteolytic digestion by host cell proteases (TMPRSS2 and furin) and internalization of the viral particles. The human cell synthesizes IFN- $\alpha$ and IFN- $\beta$ in response to viral infection. The IFN responses are induced when double stranded RNA are sensed by binding proteins. The IFN act against the infected cell and limit viral replication. The inflammatory response was triggered by the entry of virus into the cell with recruitment of $\mathrm{T}$ helper cells which produce IFN- $\gamma$ Jose described the interplay between inflammation and coagulation in COVID-19. The overproduction of proinflammatory cytokines (tumor necrosis factor (TNF), IL-6, IL-1 $\beta$ called a cytokine storm, leading to vascular hyperpermeability. There is a crosstalk between coagulation and inflammation pathways. The thrombin promote clot formation by activating platelets and converting fibrinogen to fibrin. The thrombin augments inflammation via proteinase activated receptors
(PAR). The formation of thrombin is controlled by negative feedback loops and anticoagulants, such as antithrombin III, tissue factor pathway inhibitor, and the protein $C$ system. The impairment of all the three control mechanisms cause procoagulant-anticoagulant imbalance, that predisposes to microthrombosis, DIC, multi-organ failure and death [13].

The pulmonary pathology consists of hyaline membrane formation, desquamation of pneumocytes in alveolar spaces, and an presence of lymphocytes and monocytes in interstitial space. Giant cells can be seen and Type II pneumocytes showed presence of viral particles. $[6,11]$.In a meta-analysis Aziz found IL-6 is an important proinflammatory biomarker for prediction of severe COVID-19 cases [14]. In a review, Terpos suggested prognostic value of NLR and peak PLR in determining severe cases [15]. Longitudinal evaluation of lymphocyte count and markers of inflammation like LDH, CRP and IL- 6 help in identification of cases with dismal prognosis. Biomarker, such as high serum procalcitonin and ferritin emerged as poor prognostic factors. Furthermore, hypercoagulability is correlated with adverse outcome. The elevated D-dimer levels, PT and APTT prolongation, increased FDP and severe thrombocytopenia lead to increased DIC $[15,16]$. The autopsy findings of COVID-19 patients were assessed by Dolhnikoff and confirmed the presence of pulmonary thrombosis. They found endothelial tumefaction and pulmonary megakaryocytes in the pulmonary capillaries that might be responsible for activation of the coagulation cascade [17]. In a similar study Connors demonstrated presence of multinucleated cells due to macrophage fusion in the alveoli in COVID-19 patients [18]. 
The molecular mechanism of diabetic complications involve increased mitochondrial reactive oxygen species (ROS) due to high glucose levels. The normalization of production of ROS by mitochondrial metabolic inhibitors and overexpression of uncoupling protein-1 (UCP-1) or manganese superoxide dismutase (MnSOD), prevents glucose-induced activation of protein kinase $C$ (PKC), formation of advanced glycation end products (AGEs), and accumulation of sorbitol, all of which lead to diabetic complications [4]. Ceriello reviewed the hypothesis that hyperglycemia is the key factor in hypercoagulability in diabetics. Hyperglycemia acts through three molecular mechanisms that involve nonenzymatic glycation, the development of increased oxidative stress and a decrease in the levels of heparan sulphate. Hyperglycemia cause increased levels of FI, FVII, FVIII, von Willebrand Factor and FX [19]. In a review, Alzahrani reported that the expression of tissue factor by vascular and non-vascular cells was up-regulated due to presence of low grade inflammation in type 2 diabetes mellitus [20]. Increased levels of FVII were also evident, which increases the risk of thrombus formation. As reported by others, we found increased levels of FVII and tissue factor as evidenced by isolated increase in PT.

In a study with similar findings, Abdulla compared PT and APPT in diabetics and non-diabetics and found no significant difference between these two groups [21]. Bukhari studied effects of SARS-CoV-2 on gestational diabetes mellitus. Despite lack of placental infection by SARS-CoV-2, abnormal placental morphology has been recorded. Till now, no scientific proof has been documented regarding impact of COVID-19 on pregnancy and fetus [22].

The pathophysiological interactions in COVID-19 and diabetes mellitus has been presented by Singh [6]. Several mechanisms has been emphasized other than the usual mechanism of impaired neutrophil chemotaxis and phagocytosis in diabetics, responsible for a higher mortality risk and severity of infection. The expression of ACE-2 receptors were found to be higher in the renal cortex, liver and pancreas, but not in the lungs. The significance of these observations is not clear at present, indicating ACE-2 expression may predispose diabetics to infection. Furthermore, increased furin, impaired T-Cell function, and increased interleukin-6 (IL-6) are seen in diabetics. As ACE- 2 receptors are expressed in pancreatic islets, hyperglycemia has been observed in cases of SARS-CoV-2 without pre-existing diabetes.

\section{Conclusions}

The study concludes that coagulation and fibrinolysis system is not the place of interactions in the pathogenesis of diabetes mellitus and SARS-CoV-2 infection. Though the patients' age is matched in our study, the age related changes may be one of the factor leading to higher mortality. Further explanatory studies are needed to explain the higher mortality in diabetic patients suffering from COVID-19.

\section{Limitations of study}

This is an hospital based study. The study has small sample size, therefore non-parametric analysis was performed.

\section{Conflicts of interest}

There is no conflicts of interest among authors.

\section{REFERENCES}

1. WHO Coronavirus Disease (COVID-19) Dashboard. COVID 19 Special Issue. 2020; 10(1), doi: 10.46945/bpj.10.1.03.01.

2. IDF Diabetes Atlas Ninth edition 2019, International Diabetes Federation. Available online: https://www.idf.org/aboutdiabetes/ what-is-diabetes/facts-figures.html. [Last accessed: 05.11.2020].

3. Bloomgarden ZT. Diabetes and COVID-19. J Diabetes. 2020; 12(4): 347-348, doi: 10.1111/1753-0407.13027, indexed in Pubmed: 32162476.

4. Nishikawa T, Araki E. Impact of mitochondrial ROS production in the pathogenesis of diabetes mellitus and its complications. Antioxid Redox Signal. 2007; 9(3): 343-353, doi: 10.1089/ ars.2006.1458, indexed in Pubmed: 17184177.

5. Ma X, Chen Z, Wang Le, et al. The pathogenesis of diabetes mellitus by oxidative stress and inflammation: its inhibition by berberine. Front Pharmacol. 2018; 9: 782, doi: 10.3389/ fphar.2018.00782, indexed in Pubmed: 30100874.

6. Singh AK, Gupta R, Ghosh A, et al. Diabetes in COVID-19: prevalence, pathophysiology, prognosis and practical considerations. Diabetes Metab Syndr. 2020; 14(4): 303-310, doi: 10.1016/j. dsx.2020.04.004, indexed in Pubmed: 32298981.

7. Indrayan A, Malhotra RK. Medical biostatistics. 4th ed. In: Relationships. Quantitative Outcome. CRC Press, Taylor \& Francis Group 2018: 456

8. JASP Team, JASP (version 0.12.2) [Computer Software], University of Amsterdam, Netherlands. 2020; Copyright 2013-2019.

9. MATLAB Team, Statistics and Machine Learning Toolbox 10.2, MATLAB. version 9.0.0.341360 (R 2016a). Natick, Massachusetts The Mathworks Inc.

10. Boron WF, Boulpaep EL. Medical physiology. 4th ed. Elsevier Health Sciences 2016: 439-442.

11. , et al Fauci AS, Kasper DL, Hauser SL, et al. Harrison's principles of internal medicine. : McGraw-Hill, New York 2012: 973.

12. Mann KG, Butenas S, Brummel K. The dynamics of thrombin formation. Arterioscler Thromb Vasc Biol. 2003; 23(1): 17-25, doi: 10.1161/01.atv.0000046238.23903.fc, indexed in Pubmed: 12524220.

13. Jose R, Manuel A. COVID-19 cytokine storm: the interplay between inflammation and coagulation. The Lancet Respiratory Medicine. 2020; 8(6): e46-e47, doi: 10.1016/s2213-2600(20)30216-2.

14. Aziz M, Fatima R, Assaly R. Elevated interleukin-6 and severe COVID-19: A meta-analysis. J Med Virol. 2020; 92(11): 2283-2285, doi: 10.1002/jmv.25948, indexed in Pubmed: 32343429.

15. Terpos E, Ntanasis-Stathopoulos I, Elalamy I, et al. Hematological findings and complications of COVID-19. Am J Hematol. 2020; 95(7): 834-847, doi: 10.1002/ajh.25829, indexed in Pubmed: 32282949 . 
16. Giannis D, Ziogas IA, Gianni P. Coagulation disorders in coronavirus infected patients: COVID-19, SARS-CoV-1, MERS-CoV and lessons from the past. J Clin Virol. 2020; 127: 104362, doi: 10.1016/j.jcv.2020.104362, indexed in Pubmed: 32305883.

17. Dolhnikoff M, Duarte-Neto AN, de Almeida Monteiro RA, et al. Pathological evidence of pulmonary thrombotic phenomena in severe COVID-19. J Thromb Haemost. 2020; 18(6): 1517-1519, doi: 10.1111/jth.14844, indexed in Pubmed: 32294295.

18. Connors JM, Levy JH. Thromboinflammation and the hypercoagulability of COVID-19. J Thromb Haemost. 2020; 18(7): 1559-1561, doi: 10.1111/jth.14849, indexed in Pubmed: 32302453.

19. Ceriello A. Coagulation activation in diabetes mellitus: the role of hyperglycaemia and therapeutic prospects. Diabetologia.
1993; 36(11): 1119-1125, doi: 10.1007/BF00401055, indexed in Pubmed: 8270125.

20. Alzahrani SH, Ajjan RA. Review article: Coagulation and fibrinolysis in diabetes. Diabetes and Vascular Disease Research. 2010; 7(4): 260-273, doi: 10.1177/1479164110383723.

21. Abdulla AM, Elmissbah TE, Hamid EM, et al. Assessment of coagulation process in diabetic patients using prothrombin time and activated thromboplastin time tests. Int. J. of Multidisciplinary and Current Research. 2017; 5: 343-347.

22. Bukhari M. COVID-19 in patients with gestational diabetes: review of literature. Clinical Diabetology. 2020; 9(6): 367-371, doi: DK.2020.0056. 